\title{
Radiological factors related to recurrence of chronic subdural hematoma
}

\author{
Kimihiro Nagatani • Satoru Takeuchi • \\ Fumihiro Sakakibara • Naoki Otani • \\ Hiroshi Nawashiro
}

Received: 4 February 2011 /Accepted: 7 February 2011 /Published online: 24 February 2011

(C) Springer-Verlag 2011

We read with great interest the article titled "Use of twistdrill craniostomy with drain in evacuation of chronic subdural hematomas: independent predictors of recurrence" by Escosa et al. [1]. The authors analyzed factors related to the recurrence of hematoma in 312 consecutive patients with chronic subdural hematoma (CSDH) who were treated with twist-drill craniostomy (TDC) with a drain. They measured the radiological parameters of $\mathrm{CSDH}$, namely, hematoma width, midline shift, and hematoma side, before and after the operation, and concluded that preoperative and postoperative hematoma width and midline shift are independent predictors of recurrence. We completely agree with the viewpoint of Escosa et al., and we wish to provide further comment on this issue. Nakaguchi et al. [3] reported that they classified CSDHs into four types according to the internal architecture and density of hematomas, and defined the "separated type" as a hematoma containing two components of different densities separated by a clear boundary-that is, a lower density component located above a higher density component. Among all the CSDH types, the separated type had the highest recurrence rate $(36 \%)$, as reported by some previous studies $[2,4]$. We have also reported the recurrence rates in 64 consecutive patients after $\mathrm{CSDH}$ evacuation [5]. Recurrence was noted in seven of the $64(10.9 \%)$ patients after burr-hole craniostomy with a drain, and the separated type of CSDH

K. Nagatani $(\bowtie) \cdot$ S. Takeuchi $\cdot$ F. Sakakibara $\cdot$ N. Otani $\cdot$

H. Nawashiro

Department of Neurosurgery, National Defense Medical College,

3-2 Namiki,

Tokorozawa, Saitama 359-8513, Japan

e-mail: naval.kimi@gmail.com was seen in three $(42.9 \%)$ of the seven recurrent cases. Furthermore, Nomura et al. [4] analyzed the concentrations of fibrinogen, fibrin monomer, and d-dimer in patients with "layering-type" $\mathrm{CSDH}$, which is equivalent to the separated type, and reported that the layering type of CSDH is active, has a high tendency to rebleed, and exhibits hyperfibrinolytic activity. Based on these reports, we consider that further investigation to determine the correlation of internal architecture and density of hematomas with the recurrence of CSDH may provide additional insight into the recurrence of $\mathrm{CSDH}$ after TDC with a drain.

Conflicts of interest None.

\section{References}

1. Escosa Baé M, Wessling H, Salca HC et al (2010) Use of twist-drill craniostomy with drain in evacuation of chronic subdural hematomas: independent predictors of recurrence. Acta Neurochir. doi:10.1007/s00701-010-0903-3

2. Fujioka S, Matsukado Y, Kaku M, Sakurama N, Nonaka N, Miura G (1981) CT analysis of 100 cases with chronic subdural hematoma with respect to clinical manifestation and the enlarging process of the hematoma. Neurol Med-Chir 21:1153-1160

3. Nakaguchi H, Tanishima T, Yoshimasu N (2001) Factors in the natural history of chronic subdural hematomas that influence their postoperative recurrence. J Neurosurg 95:256-262

4. Nomura S, Kashiwagi S, Fujisawa H, Ito H, Nakamura K (1994) Characterization of local hyperfibrinolysis in chronic subdural hematomas by SDS-PAGE and immunoblot. J Neurosurg 81:910-913

5. Sakakibara F, Tsuzuki N, Uozumi Y, Nawashiro H, Shima K (2011) Chronic subdural hematoma:recurrence and prevention. Brain Nerve 63:69-74 